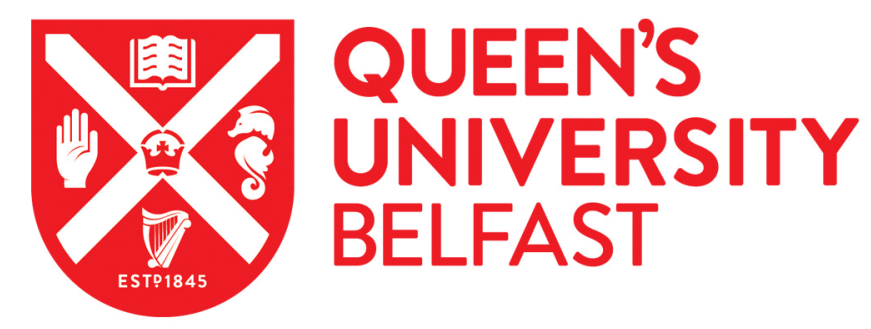

\title{
Ion Acceleration in Multispecies Targets Driven by Intense Laser Radiation Pressure
}

Kar, S., Kakolee, K. F., Qiao, B., Macchi, A., Cerchez, M., Doria, D., Geissler, M., McKenna, P., Neely, D.,

Osterholz, J., Prasad, R., Quinn, K., Ramakrishna, B., Sarri, G., Willi, O., Yuan, X. Y., Zepf, M., \& Borghesi, M. (2012). Ion Acceleration in Multispecies Targets Driven by Intense Laser Radiation Pressure. Physical Review Letters, 109(18), [185006]. https://doi.org/10.1103/PhysRevLett.109.185006

Published in:

Physical Review Letters

Document Version:

Publisher's PDF, also known as Version of record

Queen's University Belfast - Research Portal:

Link to publication record in Queen's University Belfast Research Portal

Publisher rights

(C) 2012 American Physical Society

\section{General rights}

Copyright for the publications made accessible via the Queen's University Belfast Research Portal is retained by the author(s) and / or other copyright owners and it is a condition of accessing these publications that users recognise and abide by the legal requirements associated with these rights.

Take down policy

The Research Portal is Queen's institutional repository that provides access to Queen's research output. Every effort has been made to ensure that content in the Research Portal does not infringe any person's rights, or applicable UK laws. If you discover content in the Research Portal that you believe breaches copyright or violates any law, please contact openaccess@qub.ac.uk. 


\title{
Ion Acceleration in Multispecies Targets Driven by Intense Laser Radiation Pressure
}

\author{
S. Kar, ${ }^{1, *}$ K. F. Kakolee, ${ }^{1}$ B. Qiao, ${ }^{1, \dagger}$ A. Macchi, ${ }^{2,3}$ M. Cerchez,${ }^{4}$ D. Doria, ${ }^{1}$ M. Geissler, ${ }^{1}$ P. McKenna, ${ }^{5}$ D. Neely, ${ }^{6}$ \\ J. Osterholz, ${ }^{4}$ R. Prasad, ${ }^{1}$ K. Quinn, ${ }^{1}$ B. Ramakrishna, ${ }^{1,}$ G. Sarri, ${ }^{1}$ O. Willi, ${ }^{4}$ X. Y. Yuan,${ }^{5,}$ M. Zepf,${ }^{1,7}$ and M. Borghesi ${ }^{1,8}$ \\ ${ }^{1}$ Centre for Plasma Physics, School of Mathematics and Physics, Queen's University Belfast, Belfast BT7 1NN, United Kingdom \\ ${ }^{2}$ Istituto Nazionale di Ottica, CNR, Pisa, Italy \\ ${ }^{3}$ Department of Physics "Enrico Fermi," Largo B. Pontecorvo 3, 56127 Pisa, Italy \\ ${ }^{4}$ Institut für Laser-und Plasmaphysik, Heinrich-Heine-Universität, Düsseldorf, Germany \\ ${ }^{5}$ Department of Physics, SUPA, University of Strathclyde, Glasgow G4 ONG, United Kingdom \\ ${ }^{6}$ Central Laser Facility, Rutherford Appleton Laboratory, Didcot, Oxfordshire OX11 OQX, United Kingdom \\ ${ }^{7}$ Helmholtz, Institut Jena, D-07743 Jena, Germany \\ ${ }^{8}$ Institute of Physics of the ASCR, ELI-Beamlines Project, Na Slovance 2, 18221 Prague, Czech Republic
}

(Received 23 February 2012; published 2 November 2012)

The acceleration of ions from ultrathin foils has been investigated by using $250 \mathrm{TW}$, subpicosecond laser pulses, focused to intensities of up to $3 \times 10^{20} \mathrm{~W} \mathrm{~cm}^{-2}$. The ion spectra show the appearance of narrow-band features for protons and carbon ions peaked at higher energies (in the 5-10 MeV/nucleon range) and with significantly higher flux than previously reported. The spectral features and their scaling with laser and target parameters provide evidence of a multispecies scenario of radiation pressure acceleration in the light sail mode, as confirmed by analytical estimates and 2D particlein-cell simulations. The scaling indicates that monoenergetic peaks with more than $100 \mathrm{MeV} / \mathrm{nucleon}$ are obtainable with moderate improvements of the target and laser characteristics, which are within reach of ongoing technical developments.

DOI: 10.1103/PhysRevLett.109.185006

PACS numbers: 52.38.Kd, 41.75.Ak, 41.75.Jv, 52.59.-f

Significant attention has been paid lately to laser-driven ion acceleration, which potentially offers a compact, costeffective alternative to conventional sources for scientific, technological, and health-care applications [1]. Most experimental research so far has dealt with the target normal sheath acceleration (TNSA) mechanism [1], where ions are accelerated by space charge fields set up by relativistic electrons at the target surfaces. TNSA ion beams typically have a broad energy spectrum, modest conversion efficiency at high energies, large divergence, and a $E \propto I_{0}^{1 / 2}$ $[1]$ scaling of maximum proton energy $(E)$ with peak laser intensity $\left(I_{0}\right)$. A different mechanism, radiation pressure acceleration (RPA) [2-15], is currently attracting a substantial amount of experimental and theoretical attention due to the predicted superior scaling in terms of ion energy and laser-ion conversion efficiency. In this context, the so-called "light sail" (LS) [7-15] scheme, where, for a sufficiently thin foil, the whole laser-irradiated area is detached and pushed forward by the radiation pressure, is particularly promising.

Among the attractive features of the LS mechanism are a favorable dependence on the laser fluence $[7,8,12]$, a natively narrow energy spectrum, a reduced divergence, and a similarly efficient acceleration for both protons and higher mass ions, as predicted by numerous computational and analytical studies. Experimental evidence of RPA-LS is, however, scarce, as TNSA dominates for typical interaction conditions. Henig et al. [15] have reported on spectral features, which have been associated to
RPA effects-namely, a polarization-dependent modification of the carbon ion spectral profile at moderate ion energies.

This Letter presents experimental evidence of narrowband features in the ion spectra emerging from thin foil irradiation by subpetawatt laser pulses. In particular, carbon ion peaks centered at energies up to $\sim 7 \mathrm{MeV} /$ nucleon are produced for the first time, with nearly an order of magnitude higher particle flux than previously reported [15-17]. The spectral features, and their scaling with the laser and target parameters, point to a multispecies scenario of LS acceleration as described in $[10,18]$. The possibility of achieving spectral peaks beyond $100 \mathrm{MeV} /$ nucleon, a key requirement for hadron therapy [19], by tuning currently achievable laser and target parameters, is discussed on the basis of the observed experimental scaling and supported by 2D particle-in-cell (PIC) simulations.

The experiment was carried out employing the petawatt arm of the VULCAN laser system at the Rutherford Appleton Laboratory, STFC, United Kingdom. A schematic of the experimental setup is shown in Fig. 1(a). The laser delivered $\sim 200 \mathrm{~J}$ (varying from 175 to $225 \mathrm{~J}$ in different shots) energy on target in pulses of 700-900 fs FWHM duration after being reflected off a plasma mirror $(\mathrm{PM})$, resulting in an intensity contrast ratio of $10^{9}$ between the main pulse and the nanosecond-long amplified spontaneous emission. The laser was focused on a target at normal incidence by an $f / 3$ of-axis parabolic mirror. The 


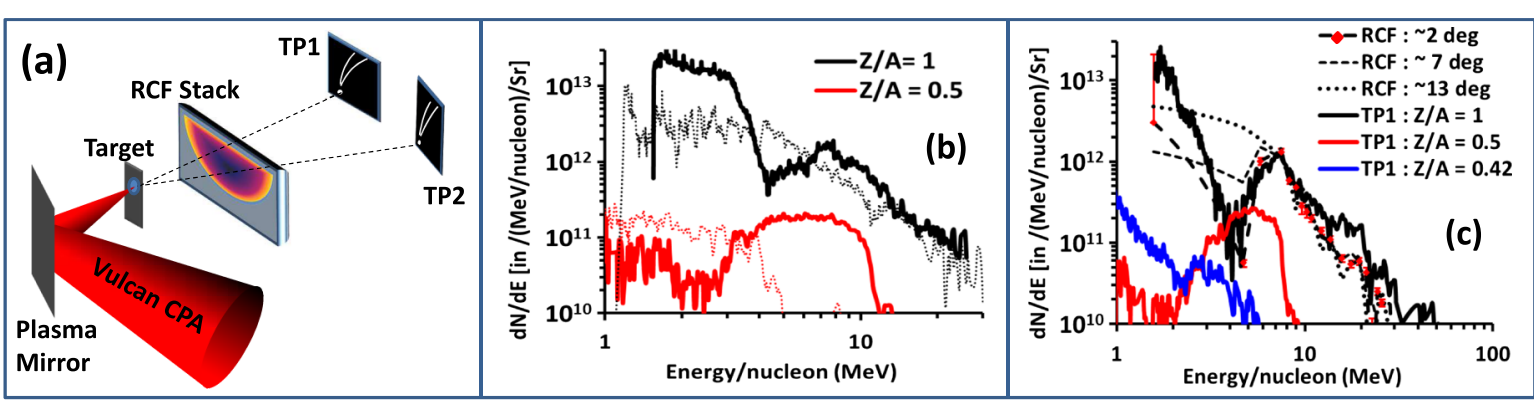

FIG. 1 (color online). (a) Schematic of the experimental setup. (b) Ion spectra obtained from $100 \mathrm{~nm}$ Cu target irradiated by a $L P$ laser pulse at $I_{0}=\sim 3 \times 10^{20} \mathrm{~W} \mathrm{~cm}^{-2}$. Different line colors correspond to different ion species (see the figure legend); solid and dotted lines represent spectra obtained on TP1 and TP2, respectively. (c) Ion spectra obtained on TP1 from $50 \mathrm{~nm}$ Cu target irradiated by a CP pulse at $I_{0} \simeq 1.25 \times 10^{20} \mathrm{~W} \mathrm{~cm}^{-2}$. The proton spectra obtained with RCF, in the same shot, at different positions in the beam, corresponding to $\sim 2^{\circ}, \sim 7^{\circ}$, and $\sim 13^{\circ}$ from the laser axis are also shown (dashed lines). RCF spectra were determined by using an iterative algorithm which calculates the spectrum yielding the closest fit to the observed dose, by evaluating the energy response function of each RCF layer and subtracting the dose contribution to a given layer resulting from ions stopping farther in the detector, similar to Ref. [21].

laser peak intensity was varied from $I_{0}=5 \times 10^{19}$ to $3 \times$ $10^{20} \mathrm{~W} \mathrm{~cm}^{-2}$ by varying the focal spot size of the laser on the target. Laser polarization on the target was varied from linear (LP) $(\varepsilon=0)$ to elliptical $(\varepsilon \sim 0.47 \pm 0.02)$ and nearly circular $(\mathrm{CP})(\varepsilon \sim 1.14 \pm 0.04)$ by employing a zero-order quarter wave plate placed between the focusing parabola and the PM. Here, $\varepsilon$ is the ratio between the laser electric field amplitudes along the vertical and horizontal axes, as determined by the combined effect of the wave plate and of reflection from the PM. Targets of different composition and thickness were irradiated. The energy spectra of the ions from the interaction were diagnosed by two Thompson parabola (TP) spectrometers as shown in Fig. 1(a) (TP1 along the laser axis and TP2 at $13 \pm 2$ degrees off axis, both with an acceptance angle of $20 \mathrm{nSr}$ ). The energy-resolved spatial profile of the bottom half of the ion beam was recorded by stacks of radiochromic films (RCF). The image plate detectors used in the TPs were cross-calibrated with CR39 solid state nuclear track detectors [20]. Similarly, the RCF dose response was absolutely calibrated [21].

While exponential spectra were always observed from 5-10 $\mu \mathrm{m}$ thick foil targets, narrow-band features in proton and heavier ion spectra were obtained from submicrometer-thick targets irradiated at high intensities. For example, the spectra in Fig. 1(b), from a $100 \mathrm{~nm}$ thick $\mathrm{Cu}$ target irradiated by a LP ( $p$ polarization) laser pulse at a peak intensity of $\sim 3 \times 10^{20} \mathrm{~W} \mathrm{~cm}{ }^{-2}$, show narrow-band peaked features in the proton (charge to mass ratio $Z / A=1)$ and carbon $(Z / A=0.5)$ spectra, clearly separated from a lower energy component (as usual in standard interaction conditions, protons and carbon ions observed in the spectrum originate from surface contaminant layers). Figure 1 (c) also shows a spectrum for $Z / A \simeq 0.42$, with a broad, exponentiallike profile. Such a charge state is likely to correspond to partially ionized $\mathrm{Cu}$, as, for instance, a close inspection suggests a fine structure within the track possibly corresponding to closely spaced values of $Z / A$. The peaks in ion spectra appear to be ordered, with the proton peak at slightly higher energy (by 2-3 MeV/nucleon) than the $\mathrm{C}$ peak. It is also interesting to note the ion flux at the peaked $\mathrm{C}$ features, which is nearly an order of magnitude higher than previously reported [15-17].

The peaks observed in carbon spectra along the laser axis are not observed in the off-axis detector TP2, as shown in Fig. 1(b), indicating that they are confined within a cone of half-aperture less than $13 \pm 2 \mathrm{deg}$. This indication is corroborated by RCF stack data taken simultaneously to the spectral measurement with the TPs [see Fig. 1(c)]. The stopping range of carbon ions is significantly shorter than for protons at the same energy per nucleon. Therefore, the deposited dose in the unsaturated RCFs, starting from the 2nd layer in the stack (which corresponds to Bragg peak energies of $4.7 \mathrm{MeV}$ and $\sim 8.5 \mathrm{MeV} /$ nucleon for protons and carbon ions, respectively), can be assumed to be primarily due to the protons. Quantitative analysis of the $\mathrm{RCF}$ indicates that the narrow-band feature in the proton spectrum detected on TP1 is observed [dashed line in Fig. 1(c)] over a defined region in the RCF corresponding to a half cone beam divergence of $\sim 10^{\circ}$. The proton spectrum gradually becomes exponential as one moves farther from the laser axis. Such exponential spectra are typical of TNSA acceleration caused by the extended sheath produced by hot electrons at the target rear surface [1]. Assuming that the divergences of the narrow-band features in the proton beam and in the carbon ion beam are comparable, the conversion efficiency into the carbon peak component can be estimated as $\sim 1 \%$, which is significantly higher than reported in [17] and comparable to Ref. [15].

The appearance and position of distinct peaks in the ion spectrum could be controlled by varying laser and target parameters as shown in Figs. 2(a) and 2(b). Peaks were 


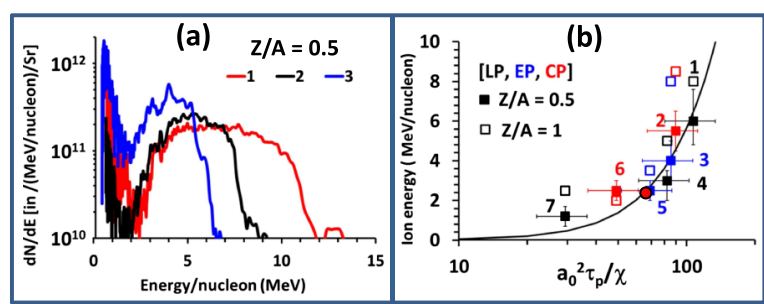

FIG. 2 (color online). (a) Graph showing comparison between three ion $(Z / A=0.5)$ spectra, where the position of the spectral peaks is plotted in (b) as a function of $a_{0}^{2} \tau_{p} / \chi$. The experimental parameter set $\left[a_{0}\right.$, target material, target thickness $\left.(\mu \mathrm{m})\right]$ for the data points $1-7$ is [15.5, $\mathrm{Cu}, 0.1],[10, \mathrm{Cu}, 0.05],[13.8, \mathrm{Cu}, 0.1]$, [7.5, Al, 0.1], [6.9, Al, 0.1], [13.6, Al, 0.5], and [14.1, Al, 0.8], respectively. The black solid line in (b) represents the ion energy estimated from the analytical modeling described in the text. The red circle represents the data from Henig et al. in Ref. [15].

observed only in the limit of thin foils and high intensity [Fig. 2(a)] with the peaks shifting towards higher energy as either the intensity was increased or the target thickness was reduced [Fig. 2(b)].

In order to assess the possible influence of radiation pressure effects on the spectral profiles observed, a simple analytical model was developed taking into account the hole boring (HB) and LS phases of the RPA mechanism. Because of the extreme radiation pressure (of the order of hundreds of GBar) exerted on the irradiated target surface, ions are swept forward by directional momentum transfer [4-6]. Consequently, the laser pulse bores through the target, in the HB phase, with the ion front velocity (in units of $c$ ) given by $[4,6,22]$

$$
\beta=\sqrt{\frac{I_{0}(t)}{\rho c^{3}}}=\sqrt{\frac{m_{e}}{m_{p}} \frac{a^{2}(t)}{\rho^{\prime}}},
$$

where $a(t)=0.85 \sqrt{I_{0}(t) \lambda^{2} / 10^{18} \mathrm{~W} \mathrm{~cm}^{-2}}, \rho^{\prime}=\rho / m_{p} n_{c}$ with $\rho$ the mass density, $m_{p}$ the proton mass, and $n_{c}$ the critical electron density, and $I_{0}(t)$ is the laser intensity on as a function of time. (Relativistic effects [6] are neglected here for simplicity, consistently with the values of $\beta \ll 1$ for our parameters.) If the target is sufficiently thin, the hole boring front will tend to reach the target rear surface at a time $t_{\mathrm{HB}}<t_{p}$ (laser pulse duration), i.e., before the end of the laser pulse. As the thickness of the compressed layer becomes comparable to or less than the evanescence length of the ponderomotive force, the whole layer can be cyclically accelerated with high efficiency for the remainder of the laser pulse duration $\left(t_{\mathrm{LS}}\right)$. In this scenario of whole foil acceleration (LS regime), the equations of motion of the foil can be expressed as [7-11]

$$
\gamma^{3} \frac{d \beta}{d \tau}=\frac{2 m_{e}}{m_{p}} \frac{1-\beta}{1+\beta} \frac{a^{2}(\tau-x)}{\chi} R, \quad \frac{d x}{d \tau}=\beta,
$$

where $\tau=c t / \lambda, \gamma=\left(1-\beta^{2}\right)^{-1 / 2}$, and $\chi=\rho^{\prime} l / \lambda$ for a target of thickness $l$, under the approximation that the areal density of the compressed layer is the same as that of the target before the interaction. The reflectivity $R=$ $R(\beta, \chi, a)$ may be estimated by using a simple model of relativistically induced self-transparency [8] and the system of equations (2) can be integrated numerically. However, some insight may already be obtained analytically. Assuming $R \simeq 1$, Eq. (2) may be integrated to yield the final velocity of the foil $\beta_{f}$ and the corresponding energy per nucleon $E_{\text {ion }}=\left(\gamma_{f}-1\right) m_{p} c^{2}$ as a function of the dimensionless fluence parameter $\mathcal{E}=\left(\rho l c^{2}\right)^{-1} \times$ $\int_{t_{0}}^{\infty} I_{0}(t) d t$ and of the initial velocity $\beta\left(t_{0}\right)=\beta_{0}[23]$ :

$$
\frac{E_{\text {ion }}}{m_{p} c^{2}}=\frac{\left(\mathcal{E}+Z_{0}\right)^{2}}{2\left(\mathcal{E}+Z_{0}+1\right)}, \quad Z_{0}=\left(\frac{1+\beta_{0}}{1-\beta_{0}}\right)^{1 / 2}-1
$$

To match the initial HB stage with the later LS one, we take $t_{0}=t_{\mathrm{HB}}$ and, from Eq. (1), $\beta_{0}=\sqrt{I\left(t_{0}\right) / \rho c^{3}}$. For thin targets and high intensities, $t_{\mathrm{HB}} \ll t_{p} \simeq t_{\mathrm{LS}}$, and the LS stage will dominate also because of higher efficiency with respect to $\mathrm{HB}$; thus, $\mathcal{E} \gg Z_{0}$. In such a limit we may estimate $\mathcal{E} \simeq a_{0}^{2} \tau_{\mathrm{LS}} / \chi \simeq a_{0}^{2} \tau_{p} / \chi$, where $\tau_{p}=c t_{p} / \lambda$ and $a_{0}^{2} \tau_{p}=\int_{-\infty}^{\infty} a^{2}(\tau) d \tau$, and $E_{\text {ion }}$ will scale as $\left(a_{0}^{2} \tau_{p} / \chi\right)^{\alpha}$ with $\alpha=2$ for $\mathcal{E} \ll 1$ and $\alpha=1$ for $\mathcal{E} \gg 1$, i.e., in the ultrarelativistic case [2].

On the basis of the above equations, we can estimate whether the observed peaks and their scaling with the target and laser parameters are consistent with the expectations for LS. The switch-on time for the LS phase is taken as the time at which the compressed ion front reaches the target rear surface as a result of the HB process. In this simple, rigid model the degree of ionization of target ions and the number of different ion species present in the compressed layer are irrelevant. Therefore, without any loss of generality, we have ignored the target contaminant layers, which have typically few nanometer thickness and significantly lower density than the metal targets used in our case. As shown in Fig. 2(b), the ion energy estimated by the model agrees well with the data (with a $R^{2}$ correlation of 0.83). As expected for the nonrelativistic case, the ion energy is seen to scale as $\left(a_{0}^{2} \tau_{p} / \chi\right)^{2}$. For comparison, the only published data point for carbon peaks attributed to RPA-LS [15] is also shown in the graph, which is in very good agreement with the calculated scaling. For the data points shown in Fig. 2(b), the hole boring phase ends significantly before the peak of the laser pulse and the nonlinear reflectivity remains at unity for the duration of the pulse.

The experimental features described above are observed independently of the incident laser polarization. This is not surprising, since the cycle-averaged light pressure is the same for CP and LP. While the early simulations of RPA were carried out by employing LP pulses at extreme intensities [2,3], the attention of theoretical work over the past few years has focused on the use of circular polarization as a means to minimize effects of electron 
heating and sheath acceleration and achieve a stable LS drive. Multidimensional simulations show that the stability of the LS acceleration is dominated by factors such as the shape of the radial laser intensity profile $[8,12]$ and achieving a smooth transition from the HB phase to the LS phase at the early stages of the acceleration process [24]. The polarization, while still affecting the outcome, plays a subordinate role in this case. One should also note that in the experiment the effect of changing the polarization of the laser pulse may be weakened by several factors, such as the polarization-dependent reflectivity of the plasma mirror (so that circular polarization actually corresponds to elliptical polarization on target with $\epsilon \sim 1.14$ ), the tight $f / 3$ focusing resulting in a spatially Gaussian intensity profile on target, and the relatively long duration of the laser pulse. The latter two factors lead to a significant deformation of the ultrathin target foil during the acceleration so that normal incidence is not strictly preserved locally. All these factors enhance electron heating and reduce any polarization dependence. Furthermore, recent work by Qiao et al. [24] has highlighted that an efficient LS drive can be achieved with LP pulses at presently achievable intensities, in a regime where RPA and TNSA coexist. In this framework, sheath-field effects may contribute to the observed separation of the $\mathrm{C}$ and proton spectral peaks [see Figs. 1(b) and 1(c)]. After the LS stage, the sheath field can in principle further accelerate the ions, with the protons gaining more energy per nucleon than carbon ions due to their higher $Z / A$. This acceleration phase may preserve or even reinforce the peaked distribution, as it typically happens in a multispecies expansion [25].

This scenario is confirmed by a 2D PIC simulation employing the ILLUMINATION code [26]. Because of the limitation in computational resources which prevents $\sim$ ps scale simulation of a long pulse interaction, we scaled down the laser and target parameters in such a way that both the dimensionless quantities $I_{0} / \rho c^{3}$ and $\left(a_{0}^{2} \tau_{p} / \chi\right)$ remain the same as in the case of Fig. 1(b). A LP laser pulse with $\lambda=1.0 \mu \mathrm{m}, I_{0}=7.5 \times 10^{19} \mathrm{~W} \mathrm{~cm}^{-2}\left(a_{0}=\right.$ 7.75), Gaussian spatial profile of $5 \mu \mathrm{m}$ radius and Gaussian temporal profile of $50 \mathrm{fs}$ FWHM was used. A copper target was used with electron density of $384.3 n_{c}$ and thickness of $l_{0}=10 \mathrm{~nm}$, covered on both sides with a $3 \mathrm{~nm}, 48.0 n_{c}$ layer of hydrocarbon contaminants. The simulation box was $20 \mu \mathrm{m}$ long and $24.576 \mu \mathrm{m}$ wide with $1 \mathrm{~nm} \times 8 \mathrm{~nm}$ cell size in order to ensure a sufficient resolution in the contaminant layers. A total of 72 particles per cell for the $\mathrm{Cu}^{28+}$ species and 36 particles per cell for $\mathrm{H}^{+}$and $\mathrm{C}^{6+}$ in the contaminant layers were used.

As expected, the simulation shows that TNSA and HB take place concurrently with the rising intensity of the laser pulse, accelerating in the forward direction ions from the rear and front surfaces, respectively. After the HB front reaches the rear surface of the target forming a compressed layer, ions are accelerated predominantly by the

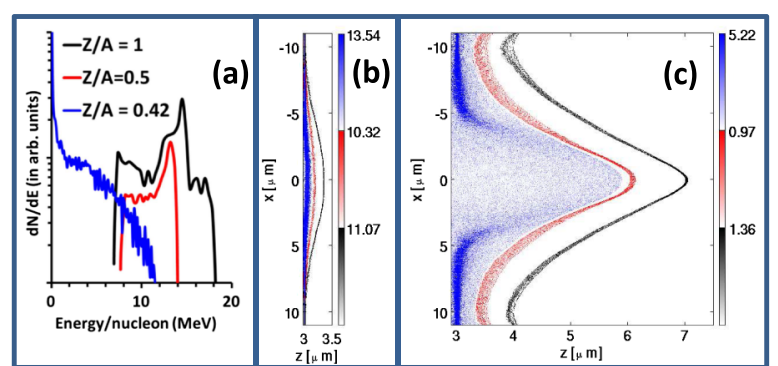

FIG. 3 (color online). (a) Ion spectra obtained from 2D multilayered PIC simulation corresponding to the data point " 1 " in Fig. 2(a) and experimental spectra of Fig. 1(b). (b) and (c) show 2D density profiles of ions at 66 and $165 \mathrm{fs}$, respectively. In all frames the $\mathrm{Cu}(Z / A=0.42), \mathrm{C}(Z / A=1 / 2)$ and proton $(Z / A=1)$ spectra (a) and distributions $(\mathrm{b}, \mathrm{c})$ are ordered from left to right. In frames $(b, c)$ each color map ranges from zero to the value shown at the top of the corresponding color bar. The color bars refer to $\mathrm{Cu}$ (top), $\mathrm{C}$ (middle), and protons (bottom), respectively.

LS mechanism resulting in the ion spectrum shown in Fig. 3(a), which reproduces the main features observed in the experimental data. The target remains highly reflective during the whole duration of the simulation, and the carbon ions are accelerated in a snowplow fashion maintaining a dense layer as shown in Figs. 3(b) and 3(c). It is interesting to note the debunching of the $\mathrm{Cu}$ ions after the laser pulse [11], as shown in Fig. 3(c), resulting in the exponential spectral profile of Fig. 3(a), which closely resembles the experimental data. This is consistent with the scenario highlighted in [10], where the heavier ions in a multispecies target undergo decompression and Coulomb explosion, while the lighter species are stabilized by the excess electrons released by the heavier species.

The experimental data points in Fig. 2(b) show that the ion energy scales quadratically with the parameter $\left(a_{0}^{2} \tau_{p} / \chi\right)$ as expected for a target with near constant reflectivity and in the limit $\beta \ll 1$. As reported by several groups via extensive 2D and 3D simulations (see Fig. 4), the ion energy can therefore be enhanced by increasing laser fluence and/or decreasing the target areal density. However, in doing this, one needs to avoid self-induced transparency, as this terminates efficient LS (see dotted and dashed lines in Fig. 4) and leads to a drastic reduction of the ion energy.

Although the ion energies achieved here $(>10 \mathrm{MeV} /$ nucleon) are encouraging, producing peaks at more than $100 \mathrm{MeV} /$ nucleon would be a crucial milestone in view of applications. As shown in the inset in Fig. 4, 2D PIC simulations predict that $100 \mathrm{MeV} /$ nucleon ions in a narrow energy bandwidth can be reached by, for example, increasing the laser fluence by a factor of 2 and decreasing the target density by a factor of 2.5 , compared to the case shown in Fig. 1(b). This seems achievable in the near term, given current developments in laser and target fabrication technology. 


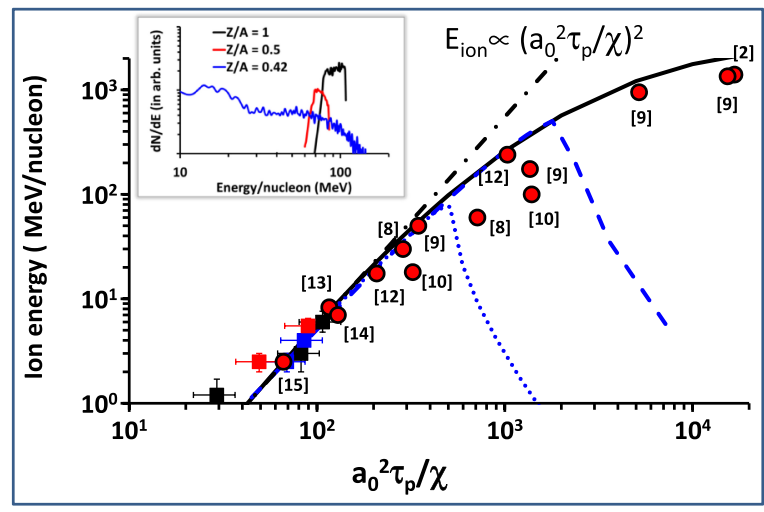

FIG. 4 (color online). Ion energy scaling (solid, black line) as shown in Fig. 2(b), extrapolated to higher $a_{0}^{2} \tau_{p} / \chi$, assuming $R=$ 1. Square points are the experimental data shown in the same figure. The (red) circles represent the spectral peaks reported in the literature, as labeled, from multispecies PIC simulations for stable LS acceleration. The dot-dashed (black) line shows the $E_{\text {ion }} \propto\left(a_{0}^{2} \tau_{p} / \chi\right)^{2}$ scaling valid for nonrelativistic ion energies. Dotted and dashed (blue) lines show the ion energy trend predicted by the rigid model by varying $\chi$ for 45 fs FWHM laser pulses at $I_{0}=5 \times 10^{20} \mathrm{~W} \mathrm{~cm}^{-2}$ and 450 fs FWHM laser pulses at $I_{0}=5 \times 10^{19} \mathrm{~W} \mathrm{~cm}^{-2}$, respectively. The inset shows the spectra obtained from a PIC simulation for a laser fluence increased by a factor of 2 and target density decreased by a factor of 2.5 with all other parameters identical to the run shown in Fig. 3.

In conclusion, we have reported on the observation of narrow-band features in the spectra of laser-accelerated ions, which appear to be consistent with radiation pressure acceleration, in a regime where LS overcomes sheath acceleration. The observed ion beams show appealing properties in terms of ion energy, divergence, and fast energy scaling and offer high promise for further progress in the near future.

The authors acknowledge funding from EPSRC [EP/ E035728/1-LIBRA consortium, EP/E048668/1 and EP/ J002550/1-Career Acceleration Fellowship held by S. K.], Leverhulme Trust (Fellowship ECF-2011-383 held by G. S.), MIUR (Italy) via the FIRB project "SULDIS," projects ELI (Grant No. CZ.1.05/1.1.00/483/02.0061) and OPVK 3 (Grant No. CZ.1.07/2.3.00/20.0279), and DFG programs TR18 and GK1203. The authors also acknowledge support from the target fabrication group and e-Science facility of RAL-STFC.

\footnotetext{
*s.kar@qub.ac.uk

†Present address: Center for Energy Research, University of California, San Diego, CA 92093-0417, USA.

${ }^{\ddagger}$ Present address: Institut für Strahlenphysik, Helmholtz Zentrum Rossendorf, Dresden, Germany.

${ }^{\S}$ Present address: Department of Physics, Shanghai Jiao Tong University, Shanghai 200240, China.
}

[1] M. Borghesi, J. Fuchs, S. V. Bulanov, A. J. MacKinnon, P. K. Patel, and M. Roth, Fusion Sci. Technol. 49, 412 (2006); H. Daido, M. Nishiuchi, and A. S Pirozhkov, Rep. Prog. Phys. 75, 056401 (2012); J. Fuchs et al., Nat. Phys. 2, 48 (2006); M. Passoni, L. Bertagna, and A. Zani, New J. Phys. 12, 045012 (2010).

[2] T. Esirkepov, M. Borghesi, S. V. Bulanov, G. Mourou, and T. Tajima, Phys. Rev. Lett. 92, 175003 (2004).

[3] T. Esirkepov, M. Yamagiwa, and T. Tajima, Phys. Rev. Lett. 96, 105001 (2006).

[4] S. C. Wilks, W. L. Kruer, M. Tabak, and A. B. Langdon, Phys. Rev. Lett. 69, 1383 (1992).

[5] S. Kar et al., Phys. Rev. Lett. 100, 225004 (2008).

[6] A. P. L. Robinson, P. Gibbon, M. Zepf, S. Kar, R. G. Evans, and C. Bellei, Plasma Phys. Controlled Fusion 51, 024004 (2009).

[7] A. Macchi, S. Veghini, and F. Pegoraro, Phys. Rev. Lett. 103, 085003 (2009).

[8] A. Macchi, S. Veghini, T. V. Liseykina, and F. Pegoraro, New J. Phys. 12, 045013 (2010).

[9] B. Qiao, M. Zepf, M. Borghesi, and M. Geissler, Phys. Rev. Lett. 102, 145002 (2009).

[10] B. Qiao, M. Zepf, M. Borghesi, B. Dromey, M. Geissler, A. Karmakar, and P. Gibbon, Phys. Rev. Lett. 105, 155002 (2010).

[11] B. Qiao, M. Zepf, P. Gibbon, M. Borghesi, B. Dromey, S. Kar, J. Schreiber, and M. Geissler, Phys. Plasmas 18, 043102 (2011).

[12] A. P. L. Robinson, M. Zepf, S. Kar, R. G. Evans, and C. Bellei, New J. Phys. 10, 013021 (2008).

[13] O. Klimo, J. Psikal, J. Limpouch, and V. T. Tikhonchuk, Phys. Rev. ST Accel. Beams 11, 031301 (2008).

[14] X. Q. Yan, C. Lin, Z. Sheng, Z. Guo, B. Liu, Y. Lu, J. Fang, and J. Chen, Phys. Rev. Lett. 100, 135003 (2008).

[15] A. Henig et al., Phys. Rev. Lett. 103, 245003 (2009).

[16] B. M. Hegelich, B. J. Albright, J. Cobble, K. Flippo, S. Letzring, M. Paffett, H. Ruhl, J. Schreiber, R. K. Schulze, and J. C. Fernández, Nature (London) 439, 441 (2006).

[17] D. Jung et al., Phys. Rev. Lett. 107, 115002 (2011).

[18] T. P. Yu, A. Pukhov, G. Shvets, and M. Chen, Phys. Rev. Lett. 105, 065002 (2010).

[19] U. Amaldi, Nucl. Phys. A654, C375 (1999); S. V. Bulanov and V. S. Khoroshkov, Plasma Phys. Rep. 28, 453 (2002).

[20] D. Doria et al., Central Laser Facility, RAL, United Kingdom, Annual Report No. 2009/10, p. 78.

[21] D. Kirkby, S. Green, F. Fiorini, D. Parker, L. Romagnani, D. Doria, S. Kar, C. Lewis, M. Borghesi, and H. Palmans, Laser Part. Beams 29, 231 (2011).

[22] A. Macchi, F. Cattani, T. V. Liseykina, and F. Cornolti, Phys. Rev. Lett. 94, 165003 (2005).

[23] J. F. L. Simmons and C. R. McInnes, Am. J. Phys. 61, 205 (1993).

[24] B. Qiao, S. Kar, M. Geissler, P. Gibbon, M. Zepf, and M. Borghesi, Phys. Rev. Lett. 108, 115002 (2012).

[25] V. Tikhonchuk, A. A. Andreev, S. G. Bochkarev, and V. Yu. Bychenkov, Plasma Phys. Controlled Fusion 47, B869 (2005), and references therein.

[26] M. Geissler, S. Rykovanov, J. Schreiber, J. Meyer-terVehn, and G. D. Tsakiris, New J. Phys. 9, 218 (2007). 